\title{
Impact of a low FODMAP diet on the amount of rectal gas and rectal volume during radiotherapy in patients with prostate cancer - a prospective pilot study
}

Christian Schaefer ${ }^{1 * \dagger}$ (D), Constantinos Zamboglou ${ }^{2,3+}$, Natalja Volegova-Neher ${ }^{2}$, Carmen Martini², Nils Henrik Nicolay ${ }^{2}$, Nina-Sophie Schmidt-Hegemann ${ }^{1}$, Paul Rogowski ${ }^{1}$, Minglun Li ${ }^{1}$, Claus Belka ${ }^{1,4}$, Arndt-Christian Müller ${ }^{5,6}$, Anca-Ligia Grosu, ${ }^{2,3}$ and Thomas Brunner ${ }^{7}$

\begin{abstract}
Background: Small inter- and intrafractional prostate motion was shown to be a prerequisite for precise radiotherapy (RT) of prostate cancer (PCa) to achieve good local control and low rectal toxicity. As rectal gas and rectal volume are known to have a relevant effect on prostate motion, this study aims to reduce these parameters by using a Low FODMAP Diet (LFD) and to show feasibility of this intervention.

Methods: We compared a prospective intervention group $(I G, n=25)$ which underwent RT for PCa and whose patients were asked to follow a LFD during RT with a retrospective control group (CG, $n=25)$ which did not get any dietary advice. In the planning CT scan and all available cone beam CT scans rectal gas was classified based on a semiquantitative score (scale from 1 to 5) and rectal volume was measured. Furthermore, patients' compliance was evaluated by a self-assessment questionnaire.
\end{abstract}

Results: Clinical and treatment characteristics were well balanced between both groups. A total of 266 (CG, 10.6 per patient) and $280 \mathrm{CT}$ scans (IG, 11.2 per patient), respectively, were analysed. The frequency distribution of gas scores differed significantly from each other $(p<.001)$ with the IG having lower scores. Rectal volume was smaller in the IG $\left(64.28 \mathrm{~cm}^{3}, 95 \% \mathrm{Cl} 60.92-67.65 \mathrm{~cm}^{3}, \mathrm{SD} 28.64 \mathrm{~cm}^{3}\right)$ than in the $\mathrm{CG}\left(71.40 \mathrm{~cm}^{3}, 95 \% \mathrm{Cl} 66.47-76.32 \mathrm{~cm}^{3}, \mathrm{SD}\right.$ $\left.40.80 \mathrm{~cm}^{3}\right)(p=.02)$. Mean intrapatient standard deviation as a measure for the variability of rectal volume was 22 $\mathrm{cm}^{3}$ in the IG and $23 \mathrm{~cm}^{3}$ in the CG $(p=.81)$. Patients' compliance and contentment were satisfying.

Conclusions: The use of a LFD significantly decreased rectal gas and rectal volume. LFD was feasible with an excellent patients' compliance. However, prospective trials with a larger number of patients and a standardized evaluation of gastrointestinal toxicity and quality of life are reasonable.

Trial registration: German Clinical Trials Register, DRKS00012955. Registered 29 August 2017 - Retrospectively registered, https://www.drks.de/drks_web/navigate.do?navigationld=trial.HTML\&TRIAL_ID=DRKS00012955

Keywords: Radiotherapy - prostate Cancer, Low FODMAP diet, Rectal volume, Rectal gas

\footnotetext{
* Correspondence: christian.schaefer@med.uni-muenchen.de

${ }^{\dagger}$ Christian Schaefer and Constantinos Zamboglou contributed equally to this

work.

'Department of Radiation Oncology, University Hospital, LMU, Marchioninistr.

15, 81377 Munich, Germany

Full list of author information is available at the end of the article
}

(c) The Author(s). 2020 Open Access This article is distributed under the terms of the Creative Commons Attribution 4.0 International License (http://creativecommons.org/licenses/by/4.0/), which permits unrestricted use, distribution, and reproduction in any medium, provided you give appropriate credit to the original author(s) and the source, provide a link to the Creative Commons license, and indicate if changes were made. The Creative Commons Public Domain Dedication waiver (http://creativecommons.org/publicdomain/zero/1.0/) applies to the data made available in this article, unless otherwise stated. 


\section{Background}

In Germany, prostate cancer (PCa) is the most common male cancer and it ranks third among the causes of cancer death in men [1]. Radiotherapy (RT) plays an important role in its treatment, both as a definitive and as a postoperative treatment option, e.g. in patients with persisting PSA values after prostatectomy or with a biochemical or local recurrence. Effectiveness of RT increases with the administered dose to the prostate $[2,3]$. However, toxicity also increases with the administered dose to the surrounding normal tissue. Thus, RT should be as exact as possible. One problem in achieving an exact RT is the positional variability of the prostate which can be divided into interfractional motion (organ motion between the fractions) and intrafractional motion (organ motion during a fraction). Prostate motion seems to be influenced relevantly by bladder filling and particularly by rectal filling [4-6]. Figure 1 shows an example for differences in rectal filling. An increased rectal volume and an increased amount of mobile rectal gas correlates with rectal movements and intrafractional motion $[7,8]$. Furthermore, a large rectal volume in the planning CT scan leads to an increased interfractional motion and consequently to a reduced local and biochemical tumour control as well as to increased gastrointestinal toxicity [9]. In recent years, non-inferiority of hypofractionated RT of PCa could be shown [10], and even ultra-hypofractionated regimens (stereotactic body radiotherapy, SBRT) were shown to be non-inferior to normofractionated RT [11]. Considering the increasing use of these new techniques with their longer time and higher dose per radiation fraction, prostate motion becomes even more important. Different approaches have been proposed to reduce prostate motion by reducing the extent and the variability of rectal volume or the amount of rectal gas during RT, including mechanical approaches like the daily application of endorectal balloons or daily enema. While the effectiveness of the endorectal balloon remains unclear, the use of daily enema seems to have a positive impact on both intraand interfractional motion [12-14]. Thus, in some radiooncology centers it is part of the clinical routine [15]. However, considering the invasive nature of these methods there still is a need for an effective and less invasive approach to reduce organ motion. Since the daily intake of a laxative, magnesium oxide, was unsuccessful $[16,17]$, the focus has shifted to dietary interventions. A number of trials already have evaluated dietary interventions during $\mathrm{RT}$ in patients with $\mathrm{PCa}$ to reduce rectal volume, rectal gas, prostate motion or gastrointestinal toxicity $[14,18-24]$. In the majority of these trials patients were asked to follow antiflatulent diets or they were instructed regarding their intake of fibres and fluids, partly in combination with laxatives. Nevertheless, despite some promising results most of these trials did not observe significant effects (see discussion for details) and none of these approaches have been implemented on a large scale in clinical routine.

In the current trial we evaluated for the first time a Low Fodmap Diet (LFD) which is usually used in patients with irritable bowel syndrome or chronic inflammatory bowel diseases. The acronym FODMAP stands
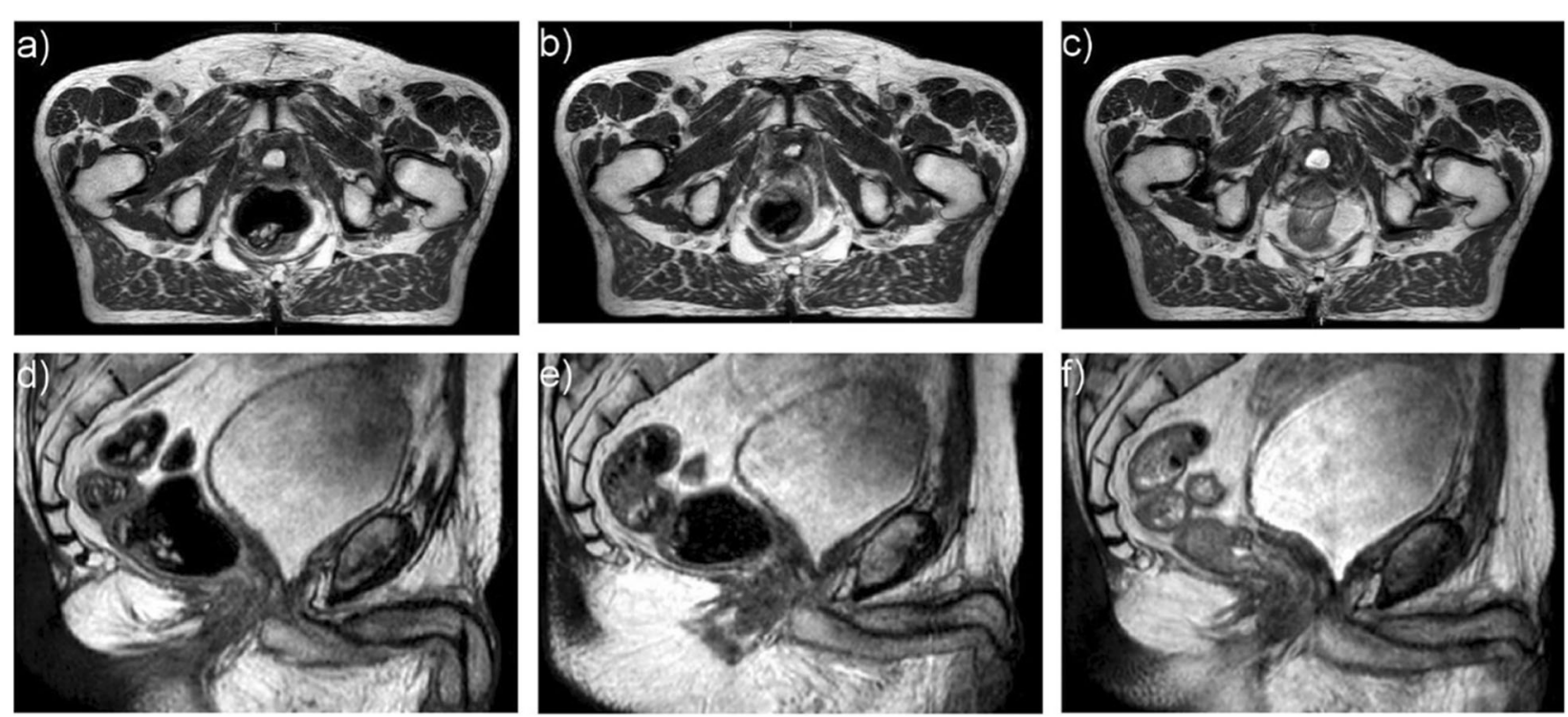

Fig. 1 T2-weighted MR sequence of a postprostatectomy patient during MR-guided salvage radiotherapy at MR Unity (Elekta ${ }^{\circledR}$. Axial (a-c) and the corresponding sagittal (d-f) views demonstrate different rectal volumes and different amounts of rectal gas during the course of fractionated radiotherapy 
for "fermentable oligo-, di- and monosaccharides and polyols". FODMAPS are short-chain carbohydrates characterized by poor absorbability, high fermentability and high osmotic potential. These characteristics lead to a large quantity of substrates in the gut, a high water influx into the intestinal lumen and a high gas production in the colon $[25,26]$. Consequently, we hypothesized that patients who follow a LFD during RT of PCa have a reduced amount of rectal gas and a smaller and less variable rectal volume compared to patients who eat normally.

\section{Methods}

The current trial was a controlled pilot study with 25 patients in each arm. A retrospective control group (CG), which did not get any dietary advice was compared to a prospective intervention group (IG), which was asked to follow a LFD during RT.

All patients were treated at the department for radiation oncology of the University Hospital Freiburg in the period from August 2014 to October 2016 (CG) and from November 2016 to January 2018 (IG), respectively. Eligible patients had to be 18 years or older, have PCa and receive a definitive, an adjuvant or a salvage RT. Patients in the IG had to sign an informed consent. Patients could not be included if they had prior rectal surgery or chronic inflammatory bowel disease, if they regularly took opioids or if RT included the pelvic lymphatic pathways. Following approval by the institutional ethics committee and registration in the German Clinical Trials Register (DRKS00012955), patients in the IG were enrolled within the frame of their first visit of the department of radiation oncology. Patients in the CG were randomly chosen by a physician who was not involved in the evaluation.

\section{Radiotherapy}

All patients were treated according to the standard operation procedure of the Department of Radiation Oncology of the University Hospital Freiburg. In the definitive setting patients received a normofractionated intensitymodulated RT (IMRT) with single doses of 2 Gy and a total dose of 74 to $78 \mathrm{~Gy}$. Adjuvant RT was performed in case of R1-resection, T3-status or a Gleason score $\geq 7 \mathrm{~b}$. In the postoperative (adjuvant or salvage) setting a normofractionated (1.8 Gy per fraction) 3D conformal RT to a dose of 14.4 to 19.8 Gy was used, followed by IMRT to a cumulative total dose of $66.6 \mathrm{~Gy}$. In case of a R1-resection or a Gleason score $\geq 8$ the total dose of adjuvant RT was escalated to $70.2 \mathrm{~Gy}$. Moreover, in case of a PET-positive local recurrence a boost to the PET-positive tumour to a total dose of 70.2 or 72 Gy was used [27]. For imageguidance, cone beam CTs were acquired before the first three fractions and after that at least weekly. Patients were asked to empty their rectum before every RT fraction and to drink $750 \mathrm{ml}$ of water $60 \mathrm{~min}$ before every RT fraction. In both groups there were no restrictions regarding the use of supportive care drugs such as macrogol in case of obstipation and simeticon in case of flatulence.

\section{Intervention}

Patients of the IG were informed about the trial at their first visit to the outpatient clinic and received a leaflet containing information about the trial and the LFD. Furthermore, they received a list with food to be avoided because of its high FODMAP concentration and possible alternative food products with a low FODMAP concentration (see Additional file 1:). The list was based on lists which had already been published $[28,29]$ and was divided into the categories "dairy products", "grain products", "fruit", "vegetables", "nuts", "legumes", "honey / syrup", "sweeteners" and "beverages" for an easy handling. Patients were asked to start with the LFD immediately after their presentation to the outpatient clinic and to follow the diet until the last day of RT. Moreover, they were asked to self-assess their adherence to the diet every day using a simple questionnaire.

\section{Data collection}

Patient data and tumour data were extracted from the electronic patient file. For contouring, Nucletron Oncentra Treatment Planning System 4.3 was used. The planning CT scan and all available cone beam CT scans were loaded and coregistered by using a mutual information registration with a clipbox. Of 560 loaded CT scans, 546 were suitable for the data collection while 14 scans could not be used due to a too small field of view or strong artefacts by hip prostheses. In both groups this corresponds to a mean number of $11 \mathrm{CT}$ scans per patient (see Table 1).

The rectal volume was calculated by contouring the external surface of the rectum in every slice $(2 \mathrm{~mm})$ from the nearest slice to the lower edge of the fourth sacral vertebra until the nearest slice to $2 \mathrm{~cm}$ cranial of the anus.

The amount of rectal gas was measured by using a five-tier semiquantitative score described by McNair et al. [21]. For evaluation, the sagittal CT slices were screened and the amount of gas was estimated and categorized (see Table 2) using the same cranial and caudal borders which have already been used for measuring the rectal volume were used.

\section{Statistical analyses}

Statistical analyses were performed with SPSS 22 and SPSS 25 (IBM). Primary endpoint was the amount of rectal gas. For comparing the groups, all CT scans were considered, in analogy to previous trials $[16,18]$. Keeping in mind the ordinal scaled data, analysis was performed with the Mann-Whitney-U-Test. Furthermore, in analogy to McNair et al. the proportion of CT scans with a score of "4" or "5" for every patient was determined [21], and 
Table 1 Patients' and treatment characteristics

\begin{tabular}{|c|c|c|c|}
\hline & $\mathrm{KG}(n=25)$ & $\mathrm{IG}(n=25)$ & statistics \\
\hline Indication & & & $p=.62^{1}$ \\
\hline primary RT & $6(24 \%)$ & $6(24 \%)$ & \\
\hline adjuvant RT & $10(40 \%)$ & $7(28 \%)$ & \\
\hline salvage RT & $9(36 \%)$ & 12 (48\%) & \\
\hline T-stage & & & $p=1.00^{2}$ \\
\hline $\mathrm{T} 2$ & $12(48 \%)$ & $11(44 \%)$ & \\
\hline $\mathrm{T} 2 \mathrm{a}$ & $4(16 \%)$ & $1(4 \%)$ & \\
\hline $\mathrm{T} 2 \mathrm{~b}$ & $1(4 \%)$ & 0 & \\
\hline $\mathrm{T} 2 \mathrm{C}$ & $6(24 \%)$ & $10(40 \%)$ & \\
\hline T2 (unspecified) & $1(4 \%)$ & 0 & \\
\hline $\mathrm{T} 3$ & $13(52 \%)$ & $13(52 \%)$ & \\
\hline T3a & $12(48 \%)$ & $10(40 \%)$ & \\
\hline T3b & $1(4 \%)$ & $2(8 \%)$ & \\
\hline T3 (unspecified) & 0 & $1(4 \%)$ & \\
\hline T4 & 0 & $1(4 \%)$ & \\
\hline N-stage & & & $p=1.00^{2}$ \\
\hline NO & $22(88 \%)$ & $23(92 \%)$ & \\
\hline N1 & $3(12 \%)$ & $2(8 \%)$ & \\
\hline M-stage & & & $p=1.00^{2}$ \\
\hline MO & $24(96 \%)$ & $24(96 \%)$ & \\
\hline M1 & $1(4 \%)$ & $1(4 \%)$ & \\
\hline Gleason-Score & & & $p=.22^{2}$ \\
\hline 6 & $2(8 \%)$ & $1(4 \%)$ & \\
\hline $7 a$ & $10(40 \%)$ & $6(24 \%)$ & \\
\hline $7 b$ & $6(24 \%)$ & $14(56 \%)$ & \\
\hline 8 & $4(16 \%)$ & $3(12 \%)$ & \\
\hline 9 & $3(12 \%)$ & $1(4 \%)$ & \\
\hline D'Amico risk & & & $p=.50^{2}$ \\
\hline low risk & $2(8 \%)$ & 0 & \\
\hline intermediate risk & $4(16 \%)$ & $6(24 \%)$ & \\
\hline high risk & $19(76 \%)$ & $19(76 \%)$ & \\
\hline ADT & & & $p=1.00^{2}$ \\
\hline yes & $4(16 \%)$ & $3(12 \%)$ & \\
\hline no & $21(84 \%)$ & 22 (88\%) & \\
\hline Age in years & & & $p=.09^{3}$ \\
\hline Mean (SD) & $69.3(6,5)$ & $65.9(7,2)$ & \\
\hline \multicolumn{4}{|l|}{ PSA in $\mathrm{ng} / \mathrm{ml}$} \\
\hline primary RT: Mean (SD) & $16.3(18.9)$ & $5.96(5.9)$ & $p=.39^{4}$ \\
\hline adjuvant / Salvage RT: Mean (SD) & $0.3(0.4)$ & $0.7(1.2)$ & $p=.31^{4}$ \\
\hline \multicolumn{4}{|l|}{ Total Dose in Gy } \\
\hline primary RT: Mean (SD) & $73.9(2.6)$ & $76(1.8)$ & $p=.14^{3}$ \\
\hline adjuvant / Salvage RT: Mean (SD) & $68.7(2.2)$ & $69.1(2.1)$ & $p=.71^{4}$ \\
\hline \multicolumn{4}{|l|}{ Usable CT scans } \\
\hline Mean (SD) & $10.6(2.0)$ & $11.2(2.6)$ & $p=.19^{4}$ \\
\hline
\end{tabular}

1: chi square test, 2: Fisher's exact test, 3: T-test, 4: Mann-Whitney-U-test
Table 2 Semiquantitative gas score by McNair et al. [21] and its frequency distribution

\begin{tabular}{llll}
\hline Score & Gas'proportion of rectal volume & CG $(n=266)$ & IG $(n=280)$ \\
\hline 1 & $0-5 \%$ & $16(6.0 \%)$ & $24(8.6 \%)$ \\
2 & $5-25 \%$ & $94(35.3 \%)$ & $131(46.8 \%)$ \\
3 & $25-50 \%$ & $73(27.4 \%)$ & $82(29.3 \%)$ \\
4 & $50-75 \%$ & $67(25.2 \%)$ & $35(12.5 \%)$ \\
5 & $75-100 \%$ & $16(6.0 \%)$ & $8(2.9 \%)$ \\
\hline
\end{tabular}

groups were compared using the Mann-Whitney-U-Test. Secondary endpoints were the rectal volume and its variability. Regarding rectal volume, groups were again compared based on all CT scans using students' t-test. In analogy to Oates et al. the standard deviation of every patient was calculated to compare the intrapatient variability of rectal volume [24]. Analysis was performed with the Mann-Whitney-U-Test. Finally, patients' compliance in the IG is reported in a descriptive way.

\section{Results}

Both groups did not differ significantly in any of the patients' or treatment characteristics (see Table 1) and thus can be well compared. No patient of the IG disrupted the LFD prematurely, so all patients and all appropriate CT scans could be used for analyses. In total, 280 CT scans in the IG (11.2 per patient) and 266 CT scans in the CG (10.6 per patient), respectively, were analysed $(p=.19)$.

\section{Rectal gas amount}

The frequency distribution of gas scores in both groups differed highly significantly from each other $(p<.001)$ with the IG having lower gas scores as is shown in Table 2. The mean proportion of CT scans with a gas score of "4" or "5" was $16 \%$ in the IG (95 CI $0.10-0.22$, SD 0.14) and $33 \%$ in the CG (95\% CI $0.21-0.44$, SD 0.27 ) and differed significantly from each other $(p=.028)$. Mean proportions of CT scans with each gas score are shown in Fig. 2.

\section{Rectal volume}

Mean rectal volume was $64.28 \mathrm{~cm} 3$ (95\% CI $60.92-$ $67.65 \mathrm{~cm} 3$, SD $28.64 \mathrm{~cm} 3)$ in the IG and $71.40 \mathrm{~cm} 3(95 \%$ CI $66.47-76.32 \mathrm{~cm} 3, \mathrm{SD} 40.80 \mathrm{~cm} 3)$ in the CG and differed significantly from each other $(p=0.02$, see Fig. 3$)$.

\section{Rectal volume variability}

Mean intrapatient standard deviation was $21.52 \mathrm{~cm} 3$ in the IG (95\% CI $15.86-27.18 \mathrm{~cm} 3$, SD $13.71 \mathrm{~cm} 3)$ and $23.44 \mathrm{~cm} 3$ in the CG $(95 \%$ CI $16.87-30.01 \mathrm{~cm} 3$, SD $15.91 \mathrm{~cm} 3)$ and did not differ significantly from each other ( $p=0.81$, see Fig. 4$)$. 


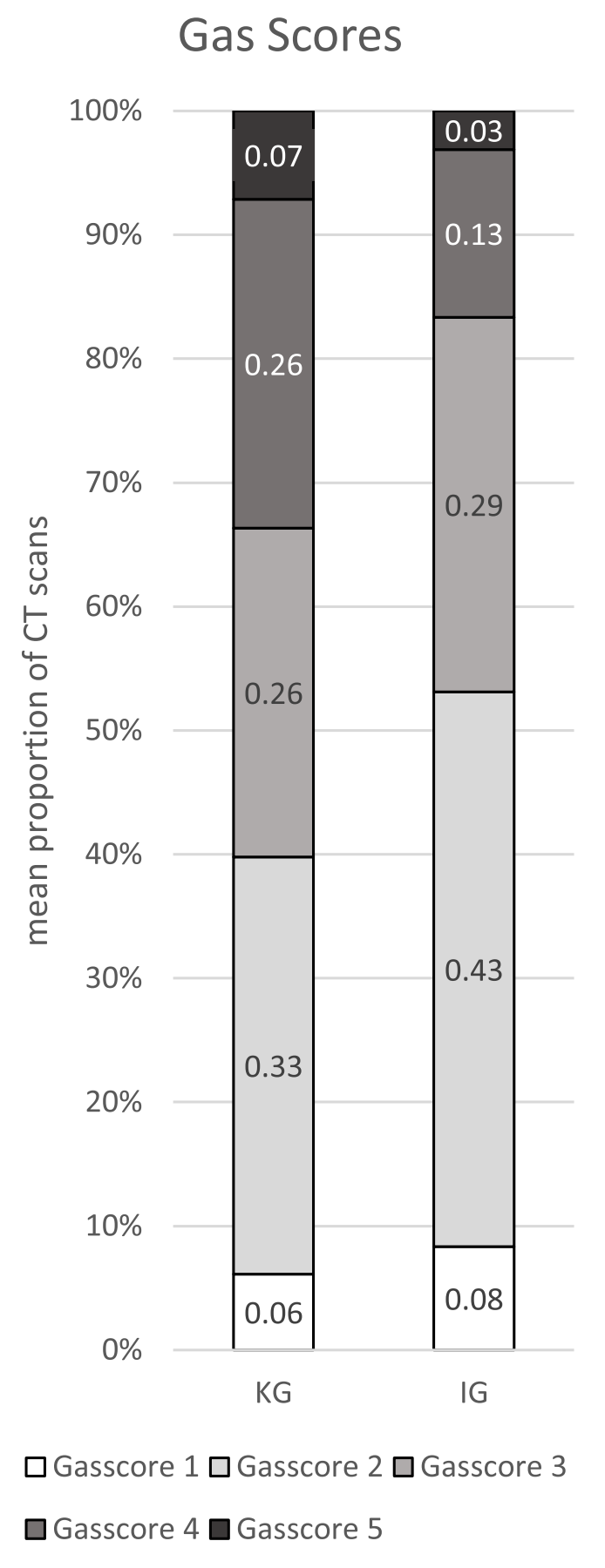

Fig. 2 Mean proportion of gas scores

\section{Compliance}

Twenty-four of 25 patients in the IG returned completely filled self-assessment questionnaires. Mean duration of the LFD and the self-assessment was 59 days (SD 6.01). Patients fulfilled the diet guidelines at $49 \%$ (95 CI $0.39-0.59$, SD 0.24 ) of the days "completely", at $39 \%$

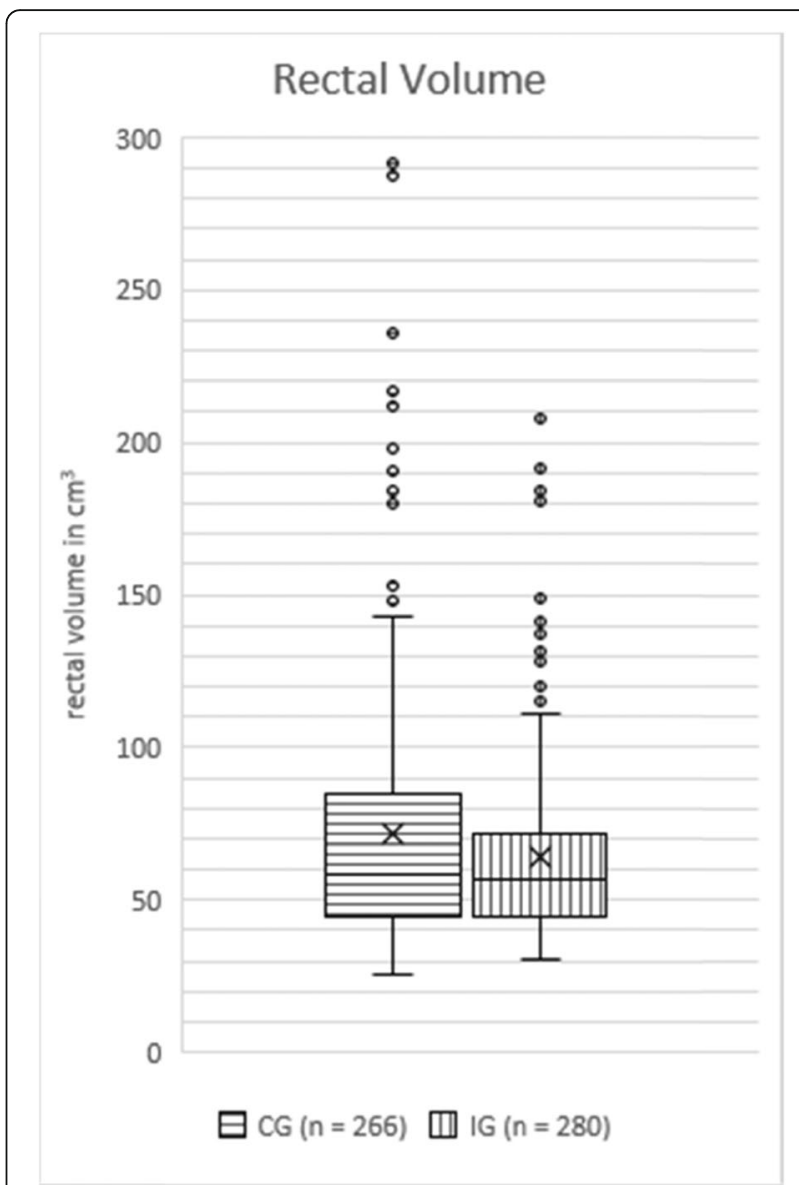

Fig. 3 Boxplot of rectal volume

(95 CI 0.31-0.47, SD 0.19) "pretty much", at 8\% (95 CI 0.05-0.11, SD 0.08) "more or less", at 5\% (95 CI 0.020.07, SD 0.06) "rather not" and at $0 \%$ (95 CI 0.00-0.01, SD 0.01) "not at all". Moreover, at the end of RT 20 of 21 patients agreed with the statement "I would recommend the LFD to a friend in the same situation" and 13 of 21 patients agreed with the statement "I could imagine following a LFD regardless of the RT". Regarding their satisfaction with digestion, 11 of 21 patients (52\%) were more content than before the treatment, 6 (29\%) reported the same degree of contentment and 4 (19\%) were less content.

\section{Discussion}

In the current pilot study, following a LFD during local $\mathrm{RT}$ of PCa led to a reduced amount of rectal gas and to a reduced rectal volume. Intrapatient variability of rectal volume did not change significantly. Overall, patients' compliance was good.

The amount of rectal gas was significantly reduced by following a LFD during RT of PCa. This corresponds with previous data showing that intake of FODMAPs leads to an increased breath hydrogen level and to a higher amount of 


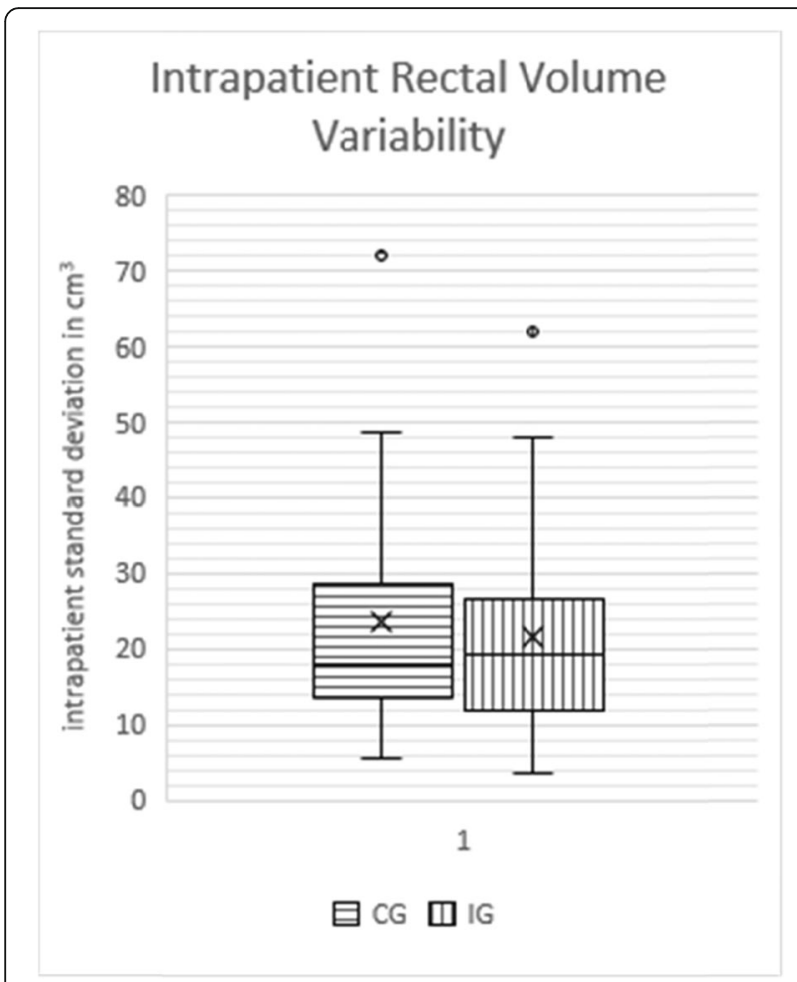

Fig. 4 Boxplot of intrapatient rectal volume variability

gas in the colon assessed by magnetic resonance imaging (MRI) [30, 31]. Taking into account that hypofractionated and ultra-hypofractionated concepts are more and more frequently employed, a reduction of intrafractional motion will be even more important in future. Thus, the result of the current pilot study is very promising as rectal gas is responsible for a relevant part of intrafractional prostate motion [7].

Rectal volume was siginificantly reduced by following a LFD as well. This also is in line with previous data which showed a reduction of stool volume and weight by a LFD in ileostomates [32]. Moreover, a MRI study showed that FODMAPs lead to a higher small bowel water content, to more colonic gas and to larger diameters of the small and large bowel with the impact depending on the subgroup of FODMAPs [31]. Hence, it will probably be helpful for future trials to distinguish further between different FODMAP subgroups in the study design. Furthermore, increasing interest in the LFD over the last years led to more extensive food lists declaring FODMAP concentrations. Thus, even better dietary advice can be given for future studies. It was observed that the full symptom-relieving effect of the LFD occurred after 7 days in patients with irritable bowel syndrome [33]. Assuming that achieving the full effect on rectal gas and volume needs a similar period of time, patients in the current trial started too late with the LFD (median 2.5 days before start of RT). There was probably just a partial effect at the time of the planning CT scan and the first few cone beam CT scans. Considering these aspects, the reduction of rectal volume in this trial already seems very encouraging.

Intrapatient variability of the rectal volume was not reduced by a LFD. To our knowledge there is no previous study evaluating this specific question. Keeping in mind the mobility of rectal gas and the reduction of rectal gas and rectal volume in the current study, one would expect a less variable rectal volume. Thus, regarding this, an evaluation with a higher sample size would be of interest. Moreover, there may be even better measures for this question than the standard deviation, such as the proportion of scans with values outside a certain range, e.g. the interquartile range.

Furthermore, we collected compliance data in the IG by using a self-assessment questionnaire. At $88 \%$ of the days patients adhered to the dietary advice "completely" or "pretty much", which in our opinion is a satisfying result. In previous studies a similarly good compliance was observed as well [33, 34]. Patients reported that the LFD was easy to implement and that the taste was good. However, LFD was slightly more expensive (10\% compared to a standard diet) and approximately one third of patients had to go to special stores to buy their food. Compliance was reduced by living together with family members or flatmates [35]. An important role in achieving the satisfying compliance probably played the high degree of satisfaction with the digestion compared to the time before start of the treatment. Furthermore, from the psychological point of view it may have had a positive impact on compliance that patients in the IG felt able to contribute to a successful treatment. Moreover, lists showing the FODMAP concentration of different food products constantly get more and more complete. Thus, it will be even easier for patients to reduce their FODMAP intake in future, which will probably improve compliance further.

First of all, the study was not randomised and not blinded due to the study design with a retrospective CG and a prospective IG. However, the design also had a positive effect as patients of the IG were not able to talk to patients of the CG and to influence their diet. On the other side, due to the study design patients with heterogenous RT concepts regarding applied doses to the target regions and RT techniques were included. Thus, despite well comparable groups regarding RT indications (see Table 1) there may have been differences in rectal doses between the groups regardless of the diet intervention. This is the reason why we did not assess any acute or chronic gastrointestinal toxicity in this study which is surely the most relevant endpoint. Further studies should evaluate also the effect of a LFD on acute and chronic gastrointestinal toxicity, which requires homogenous groups regarding dose, target volumes, RT techniques and secondary illnesses and a prospective setting with standardised methods for toxicity 
assessment. Second, the use of a semiquantitative score in combination with the missing blinding potentially could have led to a confirmation bias. Third, analogous to previous studies $[16,18]$ in this trial all CT scans $(n=546)$ were considered for analysis of rectal gas and volume. This seems reasonable as every outlier is clinically very relevant, especially during hypo- or ultrahypofractionated RT. The difference in rectal volume did not reach statistical significance when comparing patients' mean rectal volumes between the groups $(p=0.75): 64.48 \mathrm{~cm}^{3}$ in the IG (95\% CI $57.63-$ 71.34, SD $\left.16.62 \mathrm{~cm}^{3}\right)$ vs. $74.62 \mathrm{~cm}^{3}$ in the CG $(95 \% \mathrm{CI}$ $\left.58.55-90.69, \mathrm{SD} 38.94 \mathrm{~cm}^{3}\right)$. Based on the data gathered in the current study, a sample size of 196 patients would be needed to evaluate the effect of a LFD on patients' mean rectal volume (calculated with G-Power 3.1.9.2 with $\alpha=$ 0.05 and $1-\beta=0.8$ ). The frequency distribution of patients' median gas scores in both groups differed significantly from each other as well $(p=.049)$. Finally, also inflammation in the bowel may influence the rectal gas amount which was the reason for the exclusion of patients with known chronic inflammatory bowel disease. However, acute inflammatory processes in the rectum due to RT were not assessed in our study and likewise their effect on rectal gas volume is unclear.

A strength of the current study is first of all the presence of a control group whose patients could not be influenced in their diet by the intervention group due to the study design. Other positive aspects are the high number of analysed CT scans and the evaluation of patients' compliance.

Other trials evaluating dietary interventions to reduce rectal volume, rectal gas, prostate motion or gastrointestinal side effects during RT of PCa were published: Smitmans et al. (2008) tested an antiflatulent diet in combination with magnesium oxide and found a significant reduction of feces, gas and moving gas. Moreover, the success rate of 3D-grey value registration was improved significantly and there was a trend to reduced interfractional prostate motion [18]. Nichol et al. (2010) used a one-group design and cine-MRI to also evaluate an antiflatulent diet and magnesium oxide. They could not show a significant decrease in intrafractional prostate motion or the sagittal rectal area [19]. Lips et al. (2011) who also used an antiflatulent diet with magnesium oxide found a significantly increased intrafractional motion when looking at the portal images of all beams [20]. McNair et al. (2011) also used a one-group design and advised patients individually regarding their daily intake of fluids and fibres. This did not lead to a significant reduction of rectal volume. A change in rectal gas scores was the only variable correlating with rectal volume changes [21]. Pettersson et al. (2012 and 2014) asked patients to reduce their intake of insoluble fibres and lactose. There was no significant change in acute or long-term gastrointestinal side effects or other aspects of healthrelated quality of life [22, 23]. Yahya et al. (2013) used a three-group design and tested a daily microenema and a dietary intervention increasing the intake of fibres and fluids. The study showed a reduced rectal cross-sectional area and fewer geometric misses in the microenema group compared to the control group, but there was no significant effect of the diet [14]. Oates et al. (2013) evaluated an antiflatulent diet in combination with psyllium and found a trend to reduced rectal volume variability [24]. To conclude, these trials mostly did not observe significant effects and their dietary interventions have not been implemented in large scale in clinical routine.

During the time of the recruitment of the current trial a study was published evaluating the impact of the LFD in patients with gynaecological tumours receiving a RT. Compared to the control group, a better quality of life, a lower worsening of performances status during RT and a good compliance could be observed. Gastrointestinal symptoms did not change significantly [36]. Furthermore, a pilot study was published evaluating the impact of the LFD on patients with a chronic radiation enteropathy. The LFD reduced gastrointestinal symptoms significantly and led to a better quality of life [37].

To conclude, the current pilot study showed a significant reduction of rectal gas and rectal volume by the LFD. There was no difference in intrapatient rectal volume variability. Following a LFD during RT of PCa appears to be easily feasible. Hence, considering these promising results, a further prospective randomised trial with a larger sample size seems reasonable. Moreover, a further prospective evaluation should also include a standardised evaluation of gastrointestinal toxicity and patient-reported quality of life.

\section{Supplementary information}

Supplementary information accompanies this paper at https://doi.org/10. 1186/s13014-020-1474-y.

\section{Additional file 1. This list shows the nutrition advice for the dietary} intervention

\section{Abbreviations \\ 3D-CRT: Three dimensional conformal radiotherapy; CG: Control group; CT: Computer tomography; FODMAP: Fermentable oligo-, di or monosaccharides and polyols; IG: Intervention group; IMRT: Intensity modulated radiotherapy; LFD: Low FODMAP diet; PCa: Prostate cancer; PET: Positron emission tomography, RTradiotherapy; PSA: Prostate specific antigen; SBRT: Stereotactic body radiation therapy}

\section{Acknowledgements}

The piece has not been previously published and is not under consideration elsewhere. The persons listed as authors have given their approval for the submission.

\section{Authors' contributions}

CS and CZ planned and performed the study, collected and analysed the data and wrote the manuscript. NVN and CM were responsible for 
radiotherapy of most of the patients. NHN, NSSH, PR, ML, CB and ALG proofread the manuscript. ACM proofread the manuscript and contributed Fig. 1. TB suggested and planned the study, gave valuable advice during performing the study and analysing the data and edited the manuscript. All authors read and approved the final manuscript.

\section{Funding}

No funding was received.

\section{Availability of data and materials}

The dataset of the current is are available from the corresponding author on reasonable request.

\section{Ethics approval and consent to participate}

All patients gave written informed consent. This pilot study is in compliance with the principles of the Declaration of Helsinki and its subsequent amendments. This work was approved by the institutional ethics committee of the University of Freiburg.

\section{Consent for publication}

Not applicable.

\section{Competing interests}

ACM reported funding by the German Research Council (DFG) and has a research agreement with Elekta, Philips and Siemens. The other authors reported no conflicts of interest.

\section{Author details}

${ }^{1}$ Department of Radiation Oncology, University Hospital, LMU, Marchioninistr. 15, 81377 Munich, Germany. ${ }^{2}$ Department of Radiation Oncology, Medical Center, University of Freiburg, Faculty of Medicine, Freiburg, Germany. ${ }^{3}$ German Cancer Consortium (DKTK), Partner Site Freiburg, Freiburg, Germany. ${ }^{4}$ German Cancer Consortium (DKTK), Partner Site Munich, Munich, Germany. ${ }^{5}$ University Clinic for Radiation Oncology, University Hospital Tübingen, Tübingen, Germany. ${ }^{6}$ German Cancer Consortium (DKTK), Partner Site Tübingen, Tübingen, Germany. ${ }^{7}$ University Clinic for Radiation Therapy, University Hospital Magdeburg, Magdeburg, Germany.

Received: 22 November 2019 Accepted: 19 January 2020 Published online: 30 January 2020

\section{References}

1. Krebs in Deutschland für 2013/2014: 11. Ausgabe. Berlin: Robert KochInstitut and Gesellschaft der epidemiologischen Krebsregister in Deutschland e.V.; 2017

2. Dearnaley DP, Sydes MR, Graham JD, et al. Escalated-dose versus standarddose conformal radiotherapy in prostate cancer: first results from the MRC RT01 randomised controlled trial. Lancet Oncol. 2007:8:475-87.

3. Peeters STH, Heemsbergen WD, Koper PCM, et al. Dose-response in radiotherapy for localized prostate cancer: results of the Dutch multicenter randomized phase III trial comparing 68 Gy of radiotherapy with $78 \mathrm{~Gy}$. J Clin Oncol. 2006:24:1990-6.

4. Bell LJ, Cox J, Eade T, Rinks M, Kneebone A. The impact of rectal and bladder variability on target coverage during post-prostatectomy intensity modulated radiotherapy. Radiother Oncol. 2014;110:245-50.

5. Jain S, Loblaw DA, Morton GC, et al. The effect of radiation technique and bladder filling on the acute toxicity of pelvic radiotherapy for localized high risk prostate cancer. Radiother Oncol. 2012;105:193-7.

6. Zelefsky MJ, Crean D, Mageras GS, et al. Quantification and predictors of prostate position variability in 50 patients evaluated with multiple CT scans during conformal radiotherapy. Radiother Oncol. 1999;50:225-34.

7. Ghilezan MJ, Jaffray DA, Siewerdsen $\mathrm{JH}$, et al. Prostate gland motion assessed with cine-magnetic resonance imaging (cine-MRI). Int J Radiat Oncol Biol Phys. 2005;62:406-17.

8. Padhani AR, Khoo VS, Suckling J, Husband JE, Leach MO, Dearnaley DP. Evaluating the effect of rectal distension and rectal movement on prostate gland position using cine MRI. Int J Radiat Oncol Biol Phys. 1999;44:525-33.

9. de Crevoisier R, Tucker SL, Dong L, et al. Increased risk of biochemical and local failure in patients with distended rectum on the planning $C T$ for prostate cancer radiotherapy. Int J Radiat Oncol Biol Phys. 2005;62:965-73.
10. Dearnaley D, Syndikus I, Mossop $H$, et al. Conventional versus hypofractionated high-dose intensity-modulated radiotherapy for prostate cancer: 5-year outcomes of the randomised, non-inferiority, phase $3 \mathrm{CHHiP}$ trial. Lancet Oncol. 2016;17:1047-60.

11. Widmark A, Gunnlaugsson A, Beckman L, et al. Ultra-hypofractionated versus conventionally fractionated radiotherapy for prostate cancer: 5-year outcomes of the HYPO-RT-PC randomised, non-inferiority, phase 3 trial. Lancet. 2019;394:385-95.

12. Choi Y, Kwak D-W, Lee H-S, et al. Effect of rectal enema on intrafraction prostate movement during image-guided radiotherapy. J Med Imaging Radiat Oncol. 2015;59:236-42.

13. Seo YE, Kim TH, Lee KS, et al. Interfraction prostate movement in bone alignment after rectal enema for radiotherapy. Korean J Urol. 2014;55:23-8.

14. Yahya S, Zarkar A, Southgate E, Nightingale P, Webster G. Which bowel preparation is best? Comparison of a high-fibre diet leaflet, daily microenema and no preparation in prostate cancer patients treated with radical radiotherapy to assess the effect on planned target volume shifts due to rectal distension. Br J Radiol. 2013;86:20130457.

15. Nightingale H, Conroy R, Elliott T, Coyle C, Wylie JP, Choudhury A. A national survey of current practices of preparation and management of radical prostate radiotherapy patients during treatment. Radiography (Lond). 2017;23:87-93.

16. Hosni A, Rosewall T, Craig T, et al. The effect of bowel preparation regime on interfraction rectal filling variation during image guided radiotherapy for prostate cancer. Radiat Oncol. 2017;12:50.

17. Lips IM, van Gils CH, Kotte ANTJ, et al. A double-blind placebo-controlled randomized clinical trial with magnesium oxide to reduce intrafraction prostate motion for prostate cancer radiotherapy. Int J Radiat Oncol Biol Phys. 2012:83:653-60.

18. Smitsmans MHP, Pos FJ, de Bois J, et al. The influence of a dietary protocol on cone beam CT-guided radiotherapy for prostate cancer patients. Int J Radiat Oncol Biol Phys. 2008;71:1279-86.

19. Nichol AM, Warde PR, Lockwood GA, et al. A cinematic magnetic resonance imaging study of milk of magnesia laxative and an antiflatulent diet to reduce intrafraction prostate motion. Int J Radiat Oncol Biol Phys. 2010;77:1072-8.

20. Lips IM, Kotte ANTJ, van Gils CH, van Leerdam ME, van der Heide UA, van Vulpen M. Influence of antiflatulent dietary advice on intrafraction motion for prostate cancer radiotherapy. Int J Radiat Oncol Biol Phys. 2011;81:e401-6.

21. McNair HA, Wedlake L, McVey GP, Thomas K, Andreyev J, Dearnaley DP. Can diet combined with treatment scheduling achieve consistency of rectal filling in patients receiving radiotherapy to the prostate? Radiother Oncol. 2011;101:471-8.

22. Pettersson A, Johansson B, Persson C, Berglund A, Turesson I. Effects of a dietary intervention on acute gastrointestinal side effects and other aspects of health-related quality of life: a randomized controlled trial in prostate cancer patients undergoing radiotherapy. Radiother Oncol. 2012;103:333-40

23. Pettersson A, Nygren P, Persson C, Berglund A, Turesson I, Johansson B. Effects of a dietary intervention on gastrointestinal symptoms after prostate cancer radiotherapy: long-term results from a randomized controlled trial. Radiother Oncol. 2014;113:240-7.

24. Oates RW, Schneider ME, Lim Joon M, et al. A randomised study of a diet intervention to maintain consistent rectal volume for patients receiving radical radiotherapy to the prostate. Acta Oncol. 2014;53:569-71.

25. Gibson PR, Shepherd SJ. Personal view: food for thought--western lifestyle and susceptibility to Crohn's disease. The FODMAP hypothesis. Aliment Pharmacol Ther. 2005;21:1399-409.

26. Shepherd SJ, Lomer MCE, Gibson PR. Short-chain carbohydrates and functional gastrointestinal disorders. Am J Gastroenterol. 2013;108:707-17.

27. Schmidt-Hegemann N-S, Stief C, Kim T-H, et al. Outcome after PSMA PET/CT based salvage radiotherapy in patients with biochemical recurrence after radical prostatectomy: a bi-institutional retrospective analysis. J Nucl Med. 2018;60(2):227-33.

28. Gibson PR, Shepherd SJ. Evidence-based dietary management of functional gastrointestinal symptoms: the FODMAP approach. J Gastroenterol Hepatol. 2010;25:252-8.

29. Hebestreit K, Bischoff S. Das FODMAP-Konzept: Konkrete Empfehlungen für die Ernährungsberatung. Aktuel Ernahrungsmed. 2015;40:54-7.

30. Ong DK, Mitchell SB, Barrett JS, et al. Manipulation of dietary short chain carbohydrates alters the pattern of gas production and genesis of symptoms in irritable bowel syndrome. J Gastroenterol Hepatol. 2010;25:1366-73.

31. Murray K, Wilkinson-Smith V, Hoad C, et al. Differential effects of FODMAPs (fermentable oligo-, di-, mono-saccharides and polyols) on small and large intestinal contents in healthy subjects shown by MRI. Am J Gastroenterol. 2014;109:110-9. 
32. Barrett JS, Gearry RB, Muir JG, et al. Dietary poorly absorbed, short-chain carbohydrates increase delivery of water and fermentable substrates to the proximal colon. Aliment Pharmacol Ther. 2010;31:874-82.

33. Halmos EP, Power VA, Shepherd SJ, Gibson PR, Muir JG. A diet low in

FODMAPs reduces symptoms of irritable bowel syndrome.

Gastroenterology. 2014;146:67-75.e5.

34. Staudacher HM, Whelan K, Irving PM, Lomer MCE. Comparison of symptom response following advice for a diet low in fermentable carbohydrates (FODMAPs) versus standard dietary advice in patients with irritable bowel syndrome. J Hum Nutr Diet. 2011;24:487-95.

35. Gearry RB, Irving PM, Barrett JS, Nathan DM, Shepherd SJ, Gibson PR Reduction of dietary poorly absorbed short-chain carbohydrates (FODMAPs) improves abdominal symptoms in patients with inflammatory bowel disease-a pilot study. J Crohns Colitis. 2009;3:8-14.

36. Soto-Lugo JH, Souto-Del-Bosque MA, Vázquez Martínez CA. Effectiveness of a nutritional intervention in the reduction of gastrointestinal toxicity during teletherapy in women with gynaecological tumours. Revista Médica del Hospital General de México. 2018;81:7-14.

37. Larsen T, Hausken T, Otteraaen Ystad S, Hovdenak N, Mueller B, Lied GA. Does the low FODMAP diet improve symptoms of radiation-induced enteropathy? A pilot study. Scand J Gastroenterol. 2018;53:541-8.

\section{Publisher's Note}

Springer Nature remains neutral with regard to jurisdictional claims in published maps and institutional affiliations.

Ready to submit your research? Choose BMC and benefit from:

- fast, convenient online submission

- thorough peer review by experienced researchers in your field

- rapid publication on acceptance

- support for research data, including large and complex data types

- gold Open Access which fosters wider collaboration and increased citations

- maximum visibility for your research: over $100 \mathrm{M}$ website views per year

At $\mathrm{BMC}$, research is always in progress.

Learn more biomedcentral.com/submissions 